\title{
Early Findings from Hospice Service Design
}

\author{
Yang Zhao \\ School of Art and Design, Henan Institute of Engineering, Zhengzhou, Henan, China
}

\begin{abstract}
In the face of the increasing global trend of aging, hospice care has become a project that cannot be ignored in the public health field. There are many homogeneous designs with the connotation of hospice service, but clear theoretical and design principles are still vacant. This article introduces the concept of hospice service design and the development status of the current era. Focusing on the difficulties occur in research and practice process, making a brief outlook on the future development of hospice service design.
\end{abstract}

\section{1}

\section{Introduction}

The range of service design covers almost every aspect of our lives. As a user-oriented innovative thinking and method, it is widely used in business, education, medical, and public utilities. It is a must-have professional skill for modern design practitioners, and it is a systematic design solution[1] that cannot be ignored.

The beneficiaries of hospice service design are those who are defined as hopelessly treated according to current medical standards and whose life expectancy is less than 6 months. To build a complete, systematic service design requires the dual elements of both tangible and intangible design. The final delivered result is a comprehensive service package designed to improve the quality of life in the final stages of the dying. The need for hospice service design not only exists in the dying family, but also in the collective needs of the entire aging society.

The hospice service design shows the ultimate concern for life in design. It advocates that people in the dying stage can return to the mainstream, let them live as ordinary ones as much as possible, emphasizing the quality of life rather than quantity. As people become more aware of the need for hospice care, the design boundaries of related topics are also expanding. With the rapid development of mobile Internet, AI, AR and other technologies, it has a profound impact on design connotation and development trend. The hospice service design is no exception, especially in the construction of telemedicine, mobile medical, and hospice care communities, etc..

\section{The research scope of hospice service design}

The design research with the hospice spirit connotation has the following aspects:

\subsection{User research}

User research can be said as the very beginning of all modern design activities, providing strong support for theory and data. It has also played an important role in the hospice service design. The motivations, needs and expectations of the hospice phase need to be obtained at this stage. Various ethnographic study methods such as interviews, observations, focus groups, etc., and quantitative methods such as insight matrix and questionnaire are also used to collect and analyze data and information.

Of course, compared with other design projects, hospice care has a certain speciality due to the special physical and mental condition of the audience. Flexible means are needed to obtain information based on a comprehensive analysis of stakeholders (Figure 1.). For example, many dying person may not have the ability to fully express their expectations and appeals (unable to speak, unconscious etc.), so the research subject may be more inclined to other stakeholders, such as doctors and nurses with rich experience. During the process, emphasizing empathy, if researchers can participate in the immersive experience, it is more conducive to bring deep feelings and exploration of some hidden pain points. 


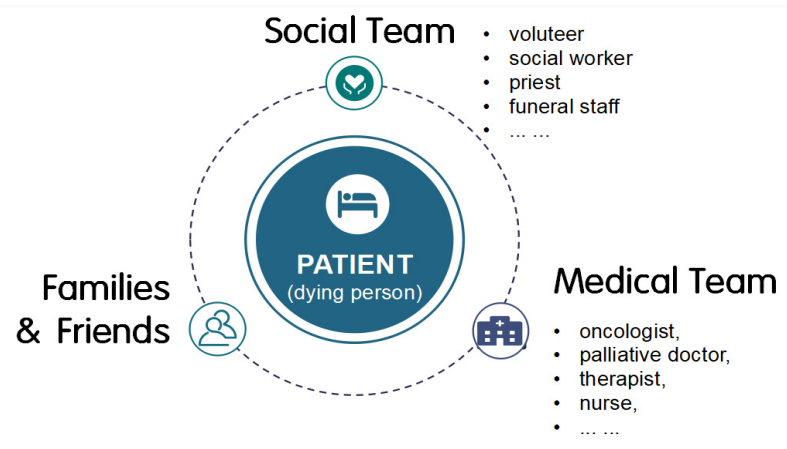

Figure 1. Stakeholders

\subsection{Tangible design}

2.1.1. Environmental planning and design. The relevant environmental planning and design as an important physical touch-point for hospice service design, including facility layout, is the most common systematic design with hospice care mind. Widely included in the design and planing of hospice care home, ward, and even extended to the funeral cemetery design.

In addition to meeting the usual industry standards and requirements, the hospice care environmental planning and design also needs to be specific to the user, and effectively consider the special needs and expectations of the dying person and their families. Through reasonable design, configuration and layout, it will achieve the lofty goal of improving the quality of life of the dying.

2.1.2. Product design. It mainly refers to those supporting products, and it has another name: rehabilitation engineering design. Rehabilitation engineering mainly refers to the science of using modern engineering theories and methods to restore compensation or reconstruct the physical function of the dying person. The hospice care products based on this include: design and development of rehabilitation assessment equipment, physical function recovery training equipment, functional reconstruction supplies (human organs, cochlear implants, etc.), body function compensation supplies (such as crutches, walkers, wheelchairs, standing frame, etc.), rehabilitation engineering materials (artificial bone joints, muscles, blood vessels, etc.), decorative pseudo-organs (artificial eyes, ears, nose, etc.). Of course, the above rehabilitation engineering design is also applicable to the rehabilitation process of other clinical patients. Hospice care products under current technology supporting also have more high-tech components, such as mobile medical platforms, wearable monitoring devices, and AI companion robots.

\subsection{Intangible design}

While design products and ideas have gradually penetrated into the hospice process, many excellent attempts have been made, but the lack of systematic design strategies, comprehensive research, and complete specification design steps have led to very limited results output. Research on the hospice service system is seen as a lean solution to the above problems. As an important part of the future hospice care process, it can not only promote the development of relevant organizations, but also actively respond to various needs and dilemmas in the implementation of hospice care.

The design process generally follows the steps below: construct a standardized, reproducible service journey map based on typical audience identity, identify the touch-points in various service processes, and cluster the pain points, so the prominent pain points will naturally be found as the opportunities for future design. Then, the design of the overall service package is carried out in a targeted manner.

\subsection{The Development of Hospice Service Design in This Internet+ Era}

In this Internet+ era, the rapid development of the technology like digital media, AI, VR and etc. have entered the various fields of public life, also had an important impact and promotion on the development of hospice service design. At present, the more representative "Internet + Hospice Care" design has the following aspects:

2.4.1. Interactive device design. Wearable device: With the continuous upgrading of core technologies such as sensors, wearable devices have played an indispensable role in the lives of users. From the "step, sleep, heart rate" monitoring gradually escalated to the social and health areas. Through the combination of software and hardware, the interaction between the user and the entire Internet of Things is expanded. By collecting and analyzing the user's physical data, prejudging the health status and giving advice on prevention or treatment, it greatly satisfies the strong demand for health management of users, especially sub-healthy urbanite.

Artificial intelligence: In the care of elderly or dying patients, there are more and more attempts to use AI robots. On the one hand, it has played an advantage in AI robots in dialogue and communication, and on the other hand, it can provide certain monitoring and reminding functions. In addition, since the functional modules can be added autonomously, in fact, the design goals of individualization are achieved. The application of nursing robots can reduce medical costs to a certain extent, standardize services, and save medical resources.

Digital interaction: The concept tombstone E-TOMB designed by students of Jiangnan University (China) uses Bluetooth technology to wirelessly make the tombstone 
"memory" and store the information about the social life of the deceased on Facebook and Twitter on the tombstone[2]. Friends and relatives can not only browse the epitome of the past life in the memory of mourning, but also continue to leave messages through the mobile terminal. It can be said that E-TOMB provides a more emotional new grave model for the Internet + era, providing a home for the deceased's online social life. This is a new way for relatives to commemorate the death of their loved ones. The design was recognized for the Red Dot Concept Award.

2.4.2. Mobile medical service. As an important means to alleviate the uneven distribution of medical resources, mobile medical care can realize many chronic disease management services. It mainly involves disease file construction and transmission, disease monitoring, audio and video consultation. In addition to the use of the remote big data platform, it also provides related help and counseling for more users.

It is worth nothing that the expansion of mobile medical service is inseparable from the popularity of mobile Internet and the development of wearable devices. The combination of soft interaction and hard interaction, online and offline, greatly ensures the effectiveness of mobile medical services.

2.4.3. Intelligent community building. We will build an integrated and intelligent community and pay attention to increase residents' awareness of health and hospice care while improving the service supporting system. The focus is on the openness and service of hospice care for all ages through the collaboration of mobile Internet, mobile terminals, telemedicine and offline medical teams, and social work organizations. Realize the participation of the whole society and eliminate the public's fear and avoidance of hospice care. In addition, the community can form stable partnerships with more external medical institutions and service groups, with higher quality hospice services as part of community identity and public policy.

\section{Difficulties in research and practice}

\subsection{User segmentation}

As an important part of the development of hospice care, the main user group of hospice care design also follows the basic service targets of hospice care and establishes both dying person and their families.

The determination of the dying person or the advanced patient is different from the usual medical science or other health care service object determination methods, neither depending on the type or system of the disease, nor based on the stage or condition of life development. That is to say, whether it is children, adolescents, youth, middle-aged or old age; no matter what kind of disease they have, as long as at the current medical level, those who have no hope of healing and are deteriorating and nearing death are considered to be dying person (late patient)[3]. The usually defined dying person is identified as having a life of no more than six months. This group of people is certainly the main target of the hospice service design (primary user group).

In addition to the importance attached to the dying person, the design of the hospice service also needs to pay attention to the families. The care and service design practice for the families also have important humanistic care value. Family members as hidden users for hospice service are often overlooked, and in fact, because the families need to face relatives in a state of sudden death or experience the loss of loved ones, they inevitably experience loss. The pain and various stress, pose a great threat to their physical and mental health. Under such circumstances, how to use design products and services to care for families, enable them to strengthen selfprotection and regulation, accept loss and adapt to new lives, is undoubtedly very important. This is in line with the biological-psychological-social-ethics design model, also the need to establish and develop a harmonious society.

\subsection{Palliative care}

Different from the curative treatment goals of traditional medicine, hospice services are all incurable dying people within 6 months of life, and are often taken care of by palliative care . WHO's definition of palliative care is " an approach that improves the quality of life of patients and their families facing the problem associated with lifethreatening illness, through the prevention and relief of suffering by means of early identification and impeccable assessment and treatment of pain and other problems, physical, psychosocial and spiritual[4]." Palliative care has played an extremely important role in today's cancer treatment and end-of-life care. It is different from laissezfaire. It regards death as a normal process neither promotes nor delays. It provides a support system so that patients can live as active as possible before death.

By clarifying the philosophy and goals of palliative care for the specificity of the users, it can help us understand more clearly the important responsibility of hospice service design in the participation and support of palliative care. The design for the dying person is no longer a display of beauty or skill, but a care design that needs to pay attention to the users' experience and truly improve the quality of their final journey. Therefore, in the design, we should avoid the rash attempt, and pursue the stable, safe and high-quality experience, which is also the principle that the hospice service design must follow.

\subsection{Pain}

Control of pain or other symptoms is a top priority in the hospice process. This cognition provides clearer guidance on the care service of the relevant workers and is also an important touch point in hospice service design. That means control of pain is a design chance point that needs special attention. 
American hospice expert Thomas Wilker specifically described the pain symptoms of late patients in the article The Model of Education for Hospice Care Services in 1991, and pointed out that the pain of dying person consists of four basic aspects, namely, physiological aspects, social aspects, emotional aspects, and spiritual aspects. The four aspects will be entangled together, causing the dying person to suffer from the pain of the whole body. In this situation, not only the person itself is miserable, but also the surrounding relatives, friends, and service workers.

After profoundly understanding the complex and special pain conditions of the dying person, how to mark the touch point that triggers the user's pain through research, and to reduce or suppress the pain as much as possible through the combination of specific products and services is of great significance. Most of the existing relevant design are designed for pain control (both for physiological and psychological) to minimize pain and improve their quality of life as a purpose.

\subsection{Psychological loss}

Psychological care is an integral part of hospice care work. End-of-life care without psychological care cannot be considered a"hospice care"in the scientific sense. Dr. Kübler Ross, a famous American psychologist, proposed five stages of pain in the psychological development of advanced patients: denial, anger, bargaining, depression and acceptance[5]. In fact, due to the different cultural backgrounds, social status, outlook on life (especially the concept of life and death), age, personality, the type of disease, the length of the disease, etc., the individual differences in psychological development and behavioral responses are also very different. Not all dying people are manifested in the psychological development as the five stages mentioned above. Even if someone has these five kinds of psychology, the order of their performance may be not the same, and even some patients may stop at a certain stage until the end of life.

In addition to the various psychological pains that the dying person themselves has to bear, the families also suffer from unprecedented pain and huge loss in the death of their loved ones. Improper handling of such loss is likely to be the root cause of certain crises. Therefore, dying psychological care not only needs to face the threat of death to the dying one, seek psychological comfort and calm, but also accompany the families to go through the entire mourning period, spend the psychological crisis period, and heal their grief caused by the huge loss.

The users of hospice service design involve groups and individuals at various levels and stages of psychological development. In order to do the corresponding design for them, the details of the condition suitable for different levels and stages should be mastered. Design with psychological care can be roughly divided into two types: one is to achieve the calm of the dying person, to control anxiety and depression; the other is to promote the mental health of the user. The former is mainly aimed at the dying person itself, while the latter is mainly concerned with the families.

\subsection{Privacy and ethics issues}

In view of the special physical and mental conditions in the final stage of life, people need to face and deal with complicated things, and many involve privacy, so there will be more obstacles in the research process. Collecting effective information(data) and demand feedback while safeguarding the privacy of the user is a major difficulty that needs to be overcome in the research process.

In addition, because it is hard to clarify the psychological endurance of patients in advanced stage, it becomes a major obstacle to the orderly development of hospice care for whether or not to clearly inform the patient of his actual condition. It involves both ethical choices and the right to know of the dying. Therefore, in the process of user research and subsequent systemic service design, the protection of user dignity and privacy, selective access to information, has become an unquestioned pain point.

\section{Conclusion}

In the context of the growing global aging crisis, how to improve the quality of life of the elderly and enable everyone to receive the respect and care they deserve at every stage of their lives is a cause that our generation needs to work for. In the past, we have gained comfort from religion and psychology. Now, we rely more on the development of material and technology. Exploring how to improve the quality of life of dying person through the cooperation of modern medicine and technology, and provide them with the best care while taking care of their dignity.

Today, hospice care is a multidisciplinary and comprehensive three-dimensional care process (Figure 1.). It pays attention to the all-round needs of the dying person and provides assistance to relatives and friends of them. It is an important component of the contemporary humanitarian cause. Our design discipline also started from its own professional characteristics and made more explorations on the hospice care cause.

Through the definition, research content and research difficulties of hospice care design, we must realize that this industry has not only prosperous development opportunities, but also serious obstacles. It requires innovation at the theoretical and technical levels, the integration of multidisciplinary knowledge. It is necessary to enhance the collective cognition of society and the participation of practitioners with humanistic care. 


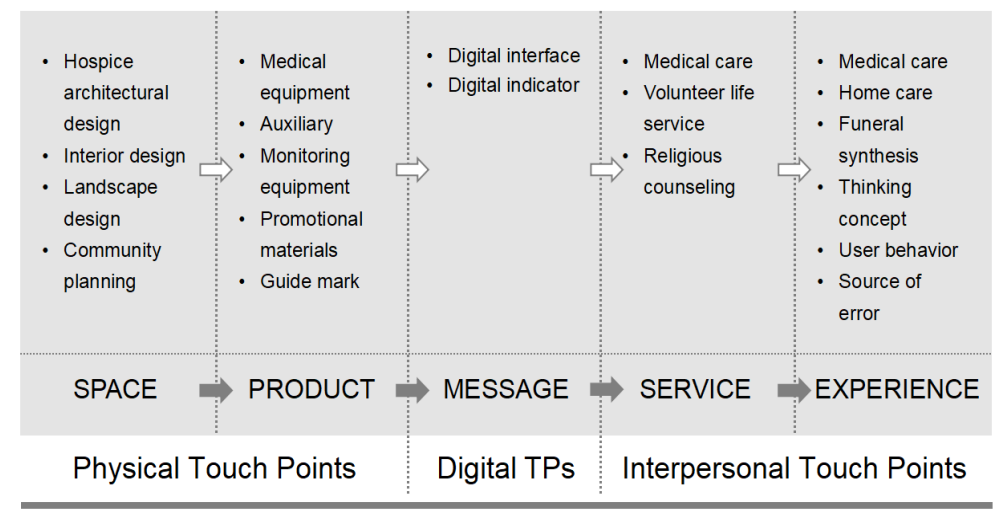

Figure 2. Touch points in hospice service design

As shown in Figure 2, it is all the design-related content in the ordinary hospice care process, also the main part of the hospice service design. It covers everything from space, products to information, services and experiences[6].

Based on the identity of the design practitioner, how to design the material and environment through the intersection and collaboration with the disciplines of medicine, psychology, sociology, mechanical engineering, rehabilitation medicine, materials science, automatic control science, artificial intelligence, etc. Planning the entire process of the care service to achieve a design care solution covering the full touch points of the service (physical touch points, digital touch points, interpersonal touch points) is undoubtedly our most ambitious goal and the most determined development direction.

\section{Acknowledgments}

This paper is the result of the "Henan Soft Science Research Project-Design and Research of Henan Rural Intelligent Medical Maintenance Platform", and the competent department is the Education Department of Henan Province.

\section{References}

1. Zhao,Y., Liu,C.C. (2020) The Lean Solution of Hospice Service Design in the "Internet Plus" Era. In: Goonetilleke,R.S., Karwowski,W., (Eds.), Advances in Physical Ergonomics and Human Factors, Springer,Washington D.C.. pp.315-326.

2. Zhao,Y. (2012) Research of Hospice Service Design, M.S. thesis. In: Dept. Design, Jiangnan Univ., Wuxi.

3. Shi,B.X. (2007) Dignity of Life and End-of-Life Care, Chongqin Press, Chongqing. pp.4-105.

4. WHO (2019) Definition of Palliative Care. https://www.who.int/cancer/palliative/definition/en/.

5. Ross,K. (2014) On Death and Dying, Yilin Press, Nanjing.

6. Ning,F., Zhu,X.J., Zhang,Y.F. (2017) Product Service Design, China Ocean University Press, Qingdao. pp32-36. 